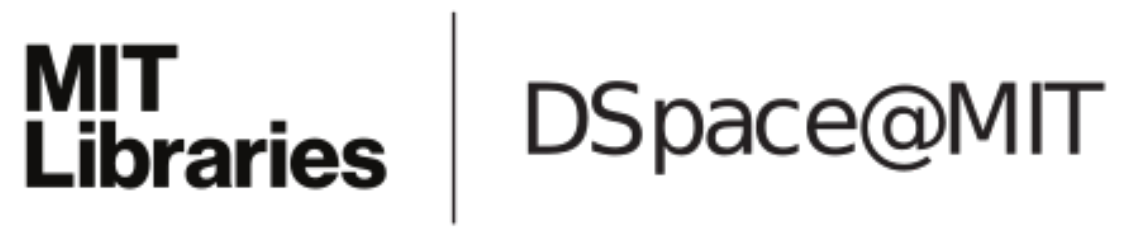

MIT Open Access Articles

Low-Flow-Coefficient Centrifugal
Compressor Design for Supercritical $\mathrm{CO}_{2}$

The MIT Faculty has made this article openly available. Please share how this access benefits you. Your story matters.

Citation: Lettieri, C. et al. "Low-Flow-Coefficient Centrifugal Compressor Design for Supercritical $\mathrm{CO}_{2}$." Journal of Turbomachinery 136, 8 (January 2014): 081008 () 2014 ASME

As Published: http://dx.doi.org/10.1115/1.4026322

Publisher: ASME International

Persistent URL: http://hdl.handle.net/1721.1/114695

Version: Final published version: final published article, as it appeared in a journal, conference proceedings, or other formally published context

Terms of Use: Article is made available in accordance with the publisher's policy and may be subject to US copyright law. Please refer to the publisher's site for terms of use. 


\section{Low-Flow-Coefficient Centrifugal Compressor Design for Supercritical $\mathrm{CO}_{2}$}

Massachusetts Institute of Technology, Cambridge, MA 02139

e-mail: lettieri@mit.edu

\author{
N. Baltadjiev \\ MIT Gas Turbine Laboratory, \\ Massachusetts Institute of Technology, \\ Cambridge, MA 02139 \\ e-mail: nikola@mit.edu
}

\author{
M. Casey \\ University of Stuttgart, \\ Stuttgart 70174, Germany \\ e-mail: casey@itsm.uni-stuttgart.de
}

Z. Spakovszky

MIT Gas Turbine Laboratory,

Massachusetts Institute of Technology,

Cambridge, MA 02139

e-mail: zolti@mit.edu
This paper presents a design strategy for very low flow coefficient multistage compressors operating with supercritical $\mathrm{CO}_{2}$ for carbon capture and sequestration (CCS) and enhanced oil recovery (EOR). At flow coefficients less than 0.01, the stage efficiency is much reduced due to dissipation in the gas-path and more prominent leakage and windage losses. Instead of using a vaneless diffuser as is standard design practice in such applications, the current design employs a vaned diffuser to decrease the meridional velocity and to widen the gas path. The aim is to achieve a step change in performance. The impeller exit width is increased in a systematic parameter study to explore the limitations of this design strategy and to define the upper limit in efficiency gain. The design strategy is applied to a full-scale reinjection compressor currently in service. Three-dimensional, steady, supercritical $\mathrm{CO}_{2}$ computational fluid dynamics (CFD) simulations of the full stage with leakage flows are carried out with the National Institute of Standards and Technology (NIST) real gas model. The design study suggests that a nondimensional impeller exit width parameter $b_{2}^{*}=\left(b_{2} / R\right) \phi$ of six yields a 3.5 point increase in adiabatic efficiency relative to that of a conventional compressor design with vaneless diffuser. Furthermore, it is shown that in such stages the vaned diffuser limits the overall stability and that the onset of rotating stall is likely caused by vortex shedding near the diffuser leading edge. The inverse of the nondimensional impeller exit width parameter $b_{2}^{*}$ can be interpreted as the Rossby number. The investigation shows that, for very low flow coefficient designs, the Coriolis accelerations dominate the relative flow accelerations, which leads to inverted swirl angle distributions at impeller exit. Combined with the twoorders-of-magnitude higher Reynolds number for supercritical $\mathrm{CO}_{2}$, the leading edge vortex shedding occurs at lower flow coefficients than in air suggesting an improved stall margin. [DOI: 10.1115/1.4026322]

\section{Introduction}

Fossil fuels account for $83 \%$ of the total US energy supply and near $90 \%$ of the worldwide primary energy source [1]. Near one third of carbon dioxide $\left(\mathrm{CO}_{2}\right)$ emissions in the US stem from coal power plants, which have an average lifespan of 35 years, and an even higher percentage is recorded worldwide. In the effort to mitigate the negative impact of the rising concentration of carbon dioxide in the atmosphere, reduction of $\mathrm{CO}_{2}$ emissions by retrofitting coal-fired power plants for carbon capture and sequestration is a high priority for research, development, demonstration, and deployment [2]. Independent of the method used to capture the $\mathrm{CO}_{2}$, central to this application is the ability to compress carbon dioxide to pressures exceeding 200 bar and to transport it in pipelines to where it is sequestered. At this pressure, the fluid reaches supercritical conditions. The compression of such a dense gas requires stages operating at flow coefficients less than 0.01 . This can demand about $15 \%$ of plant power output, dramatically increasing the plant operating costs. To enable economically viable large-scale CCS, advanced fluid machinery compatible with supercritical fluids operating at improved efficiency levels and enhanced stability margins are necessary. The high pressures and multiphase flow in these compressors and pumps yield major fluid machinery design challenges, which are the focus of the present paper.

The reasons for the poor efficiency in low flow coefficient stages are identified in the compressor and pump textbooks (for example in Aungier [3], Cumpsty [4], Gülich [5], and Lüdtke [6]). Firstly, as the design flow coefficient is reduced, the flow channels

Contributed by the International Gas Turbine Institute (IGTI) of ASME for publication in the Journal of TURBOMACHINERY. Manuscript received October 14, 2013; final manuscript received November 5, 2013; published online January 31, 2014. Editor: Ronald Bunker. become narrower and lead to higher friction losses as the proportion of end wall losses increase relative to the blade profile losses, and the hydraulic diameter of the passages is reduced. Secondly, the parasitic losses increase inversely with the flow coefficient. At a low design flow coefficient, the work input expended to overcome the disk friction of the impeller disks becomes a larger portion of the total work input, and the nonproductive leakage flow over the seals becomes a larger proportion of the impeller throughflow.

Clear guidelines about how to select a design strategy to reduce the friction losses of such stages are not given in the textbook literature. One proposal based on experimental studies of a range of stage designs is to select wider flow channels for a given flow coefficient as outlined in Casey et al. [7] and Dalbert et al. [8]. This procedure increases the hydraulic diameter but decreases the meridional velocity, so the outlet flow coefficient $c_{m} / u_{2}$ decreases and the flow angles become higher. In the design of pumps, this strategy is also adopted where it is associated with a very high impeller back sweep angle (typically $70-80 \mathrm{deg}$ ) and a reduced number of impeller blades [9]. Pumps are typically equipped with a vaned diffuser and, as a result of the very flat velocity triangle at impeller outlet, these also have a high inlet flow angle of around $80 \mathrm{deg}$. In industrial compressors, however, it is common practice to use vaneless diffusers to achieve a wide operating range. Increasing the width of the flow channels then causes high flow angles and leads to a high loss from a long flow path length in the diffuser and increased risk of rotating stall. Diffuser pinch may be used to overcome this by decreasing the flow angles, but this again leads to narrow flow channels with high losses. Due to the larger variation of density in supercritical $\mathrm{CO}_{2}$ even higher turning of the flow is observed leading to even narrower gas paths. Wider flow channels are also desired to reduce the risk of blockage due to fouling and to simplify the manufacture of the components. 
A sensible strategy to retain efficiency is to use a vaned diffuser for such stages. Some support for this strategy is given by the Aungier correlations of compressor performance [3]. For process compressors with vaneless diffusers, the efficiency is debited from that for vaned diffusers by about two points at high flow coefficients, but at a low flow coefficient this increases to four or five points. Casey et al. [7] provide experimental evidence that the short vaneless space upstream of the vaned diffuser does not appear to be a source of rotating stall. Senoo et al. [10] proposed an analytical approach to evaluate the flow behavior near instability in vaneless diffusers. The method was developed for traditional centrifugal compressors with flow coefficients much higher than in the present work. Furthermore, the approach does not capture the flow behavior near the end wall at low Rossby numbers. However, recent work in highly-loaded vaned diffusers shows that short-wavelength rotating stall precursors, so called "spikes," can occur when the spanwise flow angle profile at impeller exit is highly skewed. The radially reversed flow near the end wall induces diffuser leading edge vortex shedding, which leads to fullscale instability [11].

\section{Scope of Paper}

The review of previous work indicates that a sensible design practice for low flow coefficient stages is to select an impeller with a wider flow channel and to make use of vaned diffusers. The centrifugal compressor design for low flow coefficients then becomes similar to that of centrifugal pumps. A useful parameter is the ratio of the nondimensional impeller outlet width to the flow coefficient

$$
b_{2}^{*}=\left(b_{2} / R\right) / \phi
$$

which is, of course, directly related to the outlet flow coefficient of the stage, $c_{m} / U$. Typical high flow coefficient stages use a value of between 1 and 1.5, and designs reported by Casey et al. [7] for a flow coefficient of 0.01 and lower used a ratio of around 5 to 6 . The design rules given by Gülich [5] for pumps would suggest that a value of 10 can be used.

This paper seeks to rigorously define the limits of this strategy through an in-depth parametric study varying the nondimensional impeller exit width. It will be shown that a vaned diffuser design with a nondimensional impeller exit width of $b_{2} / R=6 \phi$ can increase the adiabatic efficiency by 3.5 points relative to that of a conventional compressor design with vaneless diffuser. Furthermore, the inverse of the nondimensional impeller exit width parameter $b_{2}^{*}$ gives a measure of the ratio of relative flow accelerations to Coriolis accelerations, the Rossby number. In supercritical $\mathrm{CO}_{2}$ compressors, the Reynolds number is 2 orders of magnitude larger than in traditional centrifugal compressor applications. This combined with the low Rossby number leads to inverted swirl angle distributions potentially improving the stable flow range.

\section{Design Considerations}

High fidelity three-dimensional CFD calculations were carried out on the first stage of the high-pressure block in a 200 bar supercritical $\mathrm{CO}_{2}$ reinjection compressor train currently in service. The stage consists of a shrouded impeller, a vaneless diffuser, a crossover bend, and a return channel with deswirl vanes. The stage inlet pressure is 68 bar and the machine Mach number is 0.75 . Single passage calculations with real gas models and leakage flows were used to dissect the loss mechanisms and to devise potential design improvements. It was found that leakage and disk friction losses account for nearly $43 \%$ of the overall losses. The largest entropy changes occur near the low-pressure labyrinth seal and near the impeller exit in the hub-side high-pressure leakage path as illustrated in Fig. 1.

The losses are associated with flow mixing and windage generated in the cavities filled with dense $\mathrm{CO}_{2}$ gas. The seal and disk

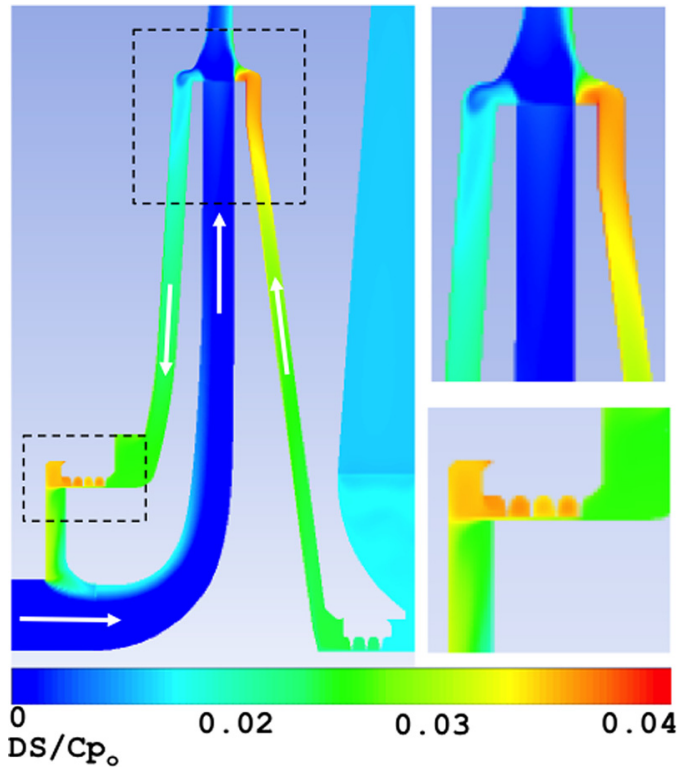

Fig. 1 Pitchwise averaged entropy change in meridional plane: seal leakage mixing losses and disk friction losses account for nearly half of the overall loss

clearances and number of labyrinth seals are in line with best practices, and the computed leakage mass flow rates agree with values reported in the literature [5] leaving little room for improvement. As such, nearly $57 \%$ of the overall losses are due to aerodynamic losses in the gas path. This suggests that further improvements in performance might be achieved through careful aerodynamic redesign of the turbomachinery components. More specifically, the strategy pursued here is to employ a vaned diffuser and, therefore, wider gas path as described above.

The work described here is focused on the redesign of a conventional low flow coefficient stage with a three-dimensional impeller and a vaneless diffuser. The new stage design consists of an impeller with prismatic, two-dimensional blades ${ }^{1}$. The pinch at the inlet to the vaned diffuser is removed so that the downstream gas path has the same width as the impeller, as shown in Fig. 2. Based on rotordynamic stability considerations for high-pressure $\mathrm{CO}_{2}$ compressors, the axial length of the stage is shortened and, for the same wheel diameter, the impeller hub-to-tip radius ratio is reduced to allow a thicker shaft. The crossover bend is of simple circular shape with constant passage width. Given the large radius of curvature relative to the passage width, the Rothstein number $r / b \cos (\alpha)$ is about 3, and flow separation is not likely (for more details see Japikse [12]). The return channel contains highly turning, front loaded vanes to deswirl the flow before it enters the next stage. The swirl vanes are carefully designed so as to mitigate potential flow separation and, therefore, flow blockage.

\section{Modeling Approach}

The design techniques are based on commercially available software tools from PCA Engineers Limited and ANSYS Inc. These have been published in detail in other sources, and so only a rudimentary description is given here. The initial estimates of the geometry were followed by more detailed design calculations using a throughflow method, known as Vista TF, which provides a model of the whole stage also including real gas effects where necessary [13]. A parameterized geometry definition system, Vista GEO, similar to that described by Casey [14] was used to define the stage component geometry. These steps were followed

${ }^{\mathrm{I}}$ Careful analysis of the flow field shows that three-dimensional effects in the relatively narrow impeller passage are negligible. 
Conventional Design

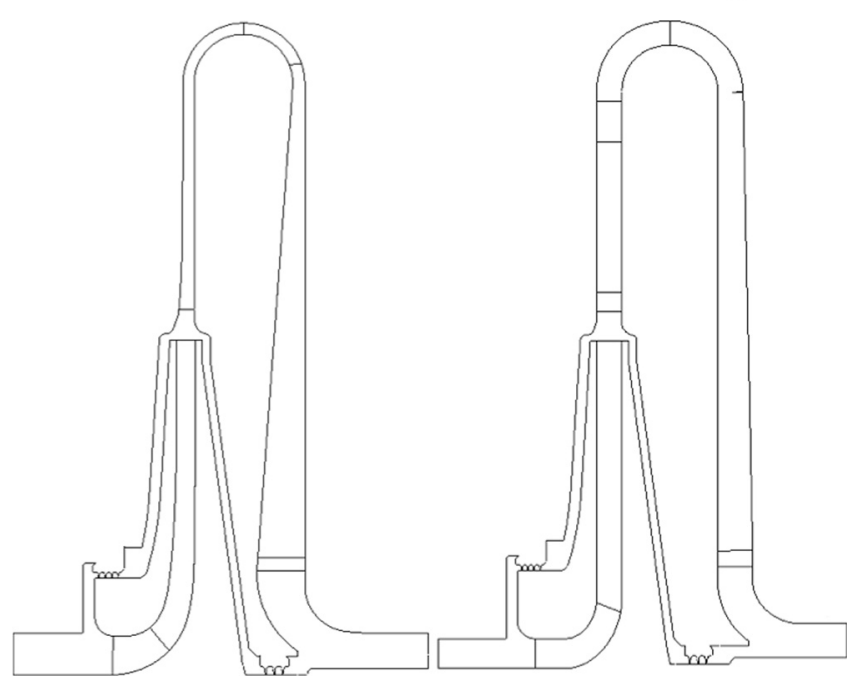

Fig. 2 Conventional gas path design with pinched vaneless diffuser (left) versus new design concept with wider gas path and vaned diffuser (right)

by a final design optimization by means of full 3D CFD simulations.

\section{Computational Method}

The computational method [15] is based on a finite-volume approach using an implicit compressible formulation with second order spatial discretization. The Reynolds-averaged NavierStokes (RANS) equations are closed through the two-equation $k-\omega$ shear stress transport turbulence model. Steady calculations are used in the parametric study and the assessment of the stage performance with and without leakage flow. The mixing plane approach [15] is used between stationary and rotating components. Unsteady RANS simulations with a second order backward Euler approach are carried out in isolated vaned diffuser calculations to assess the compressor stability.

Due to the large variations in thermodynamic properties of the supercritical $\mathrm{CO}_{2}$, special care must be taken in maintaining numerical stability. The location of the inlet and exit boundaries were chosen so as to avoid any boundary interference. In the unsteady calculations, refined time steps were required to maintain the acoustic CFL number close to unity. The total pressure was defined at the inlet boundary with the flow velocity normal to the boundary. In the stability analysis of the isolated vaned diffuser, the inlet boundary was set at the impeller exit radius and nonuniform spanwise distributions of total pressure, total temperature, and velocity components were used as described in Ref. [11]. The diffuser inlet flow profiles were taken from single stage steady isolated impeller calculations. For all the computations, the mass flow was specified at the outlet.

The wall roughness was set to an equivalent sand grain roughness of $0 \leq h_{s}^{+} \leq 5$, representative of a highly polished smooth surface. For all calculations the average $y^{+}$value was kept close to unity with at least 12 mesh cells in the boundary layer. The maximum $y^{+}$was about 20 , which was reached only in regions with large local overspeed such as near the impeller leading edge. The total number of grid points for a single passage full stage calculation amounted to $7 \times 10^{6}$ and $11 \times 10^{6}$ with and without leakage flow, respectively.

NIST's real gas equation of state (EOS) model, RefProp [16], was used in the CFD simulations. Instead of computationally costly nonlinear descriptions, the EOS model was implemented in the form of lookup tables. The real gas model was based on the Span and Wagner equation of state [17], and its implementation was tested and validated through a systematic refinement of the lookup table and comparison with measurements. It was found that for the pressure and temperature range of interest, there was no appreciable difference in compressor adiabatic efficiency and pressure coefficient if the table entries were resolved below $0.1 \mathrm{~K}$ in temperature and 0.1 bar in pressure.

Due to the supercritical fluid state, the stage Reynolds number based on wheel speed [13], $\operatorname{Re}=\Omega R b_{2} / \nu$, was of order $10^{7}$ and careful grid refinements near the end walls and blade boundaries were required to accurately capture the friction losses. Grid convergence was achieved demonstrating less than $1 \%$ change in velocity, pressure, and temperature profiles, and no appreciable difference in adiabatic efficiency and pressure coefficient.

\section{Parametric Impeller Width Study}

Single passage steady CFD calculations of the isolated impeller were first carried out in a parametric study. The objective was to determine the largest useful increase in nondimensional impeller width parameter $b_{2}^{*}$ and to quantify the performance gains and limitations. In the present study, this parameter was varied from values representing conventional, low flow coefficient centrifugal compressor designs with a $b_{2}^{*}$ of 4 to values typically used in pump designs with a $b_{2}^{*}$ of 10.5 .

In this assessment, three effects must be taken into account. First, the impeller outflow becomes more nonuniform across the span as the passage is widened, increasing the turbulent dissipation loss through the entire stage as discussed in Ref. [9]. Second, a wider flow passage yields reduced meridional velocities at impeller exit and higher flow angles into the vaned diffuser. For highly swirling flows, the distance traveled by the flow in the vaneless space is increased, thereby leading to higher friction losses. Finally, as the passage width is increased the flow becomes more tangential at the impeller exit, increasing the risk of radially reversed flow and reducing the stability margin of the diffuser.

The design constraints that need to be met in the present analysis include the required work input coefficient, flow coefficient, and machine Mach number. The blade number, the impeller outlet width, and the blade outlet angle cannot be varied independently. In particular, a larger outlet width with a constant blade outlet angle would lead to an increased head coefficient. To maintain constant work input, the blade outlet angle was varied using 1D analysis and the Wiesner slip correlation. Isolated impeller CFD simulations were then carried out using the estimated blade metal angle. The dependence of mass averaged flow angle at impeller exit on nondimensional impeller width is shown in Fig. 3 on the right.

For conventional impeller designs, the flow angle increases monotonically, starting from about $76 \mathrm{deg}$ up to about $79 \mathrm{deg}$ for $b_{2}^{*}=6$. For even larger nondimensional impeller width parameters, the flow is close to tangential, which makes the diffuser design more challenging and can reduce the stable compressor operating range. This is discussed in more detail later.

The effect on nondimensional impeller width parameter on total-to-total polytropic impeller efficiency is also evaluated in Fig. 3. The real gas polytropic efficiency was determined following the method by Schultz [18]. The results indicate that for $4 \leq b_{2}^{*} \leq 8.5$, the efficiency is insensitive to changes in nondimensional impeller width parameter. However, for $b_{2}^{*}>8$ a drop in efficiency is observed due to increased mixing losses and deteriorated impeller blade loading distributions. The latter can be mitigated by reducing the blade count as indicated by the open symbols. To estimate the diffuser performance ${ }^{2}$, Denton's $u$-cubed profile loss model [19] was applied.

${ }^{2}$ Full stage CFD computations conducted after this parametric impeller study indicate that the trend in overall vaned diffuser loss is well captured using the scaling law for profile loss. 


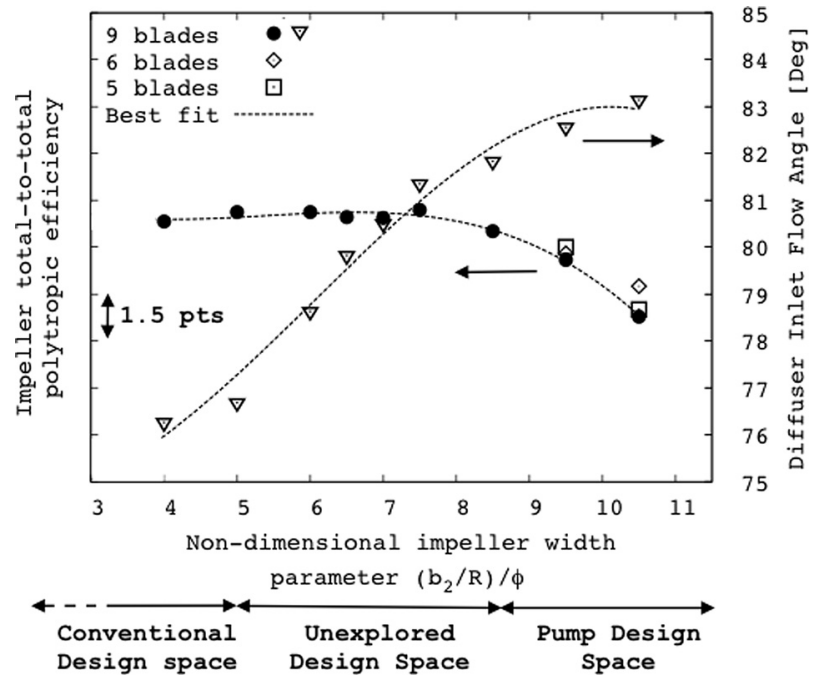

Fig. 3 Effect of $b_{2}^{*}$ on mass averaged diffuser inlet flow angle and total-to-total polytropic impeller efficiency

$$
\omega_{d}=2 C_{d} \frac{L}{W} \sum_{\text {all surfaces }} \int_{0}^{x \text { final }}\left(\frac{u_{E}}{u_{1}}\right)^{3} d\left(\frac{x}{L}\right)
$$

Here, the dissipation coefficient $C_{d}$ was chosen to be 0.002 , a typical value for turbulent boundary layers, and $L$ and $W$ are the vaned diffuser chord and passage width, respectively. For the integrand, an idealized triangular velocity distribution was assumed with the boundary layer edge velocity estimated from the isolated impeller calculations.

In Fig. 4, a large reduction in estimated diffuser loss is observed as $b_{2}^{*}$ increases to 7.5 . For even wider impellers, the reduction is dramatically diminished, and for $b_{2}^{*} \geq 8.5$ the additional gain in efficiency is relatively small. The vaneless space loss coefficient in Fig. 4 was determined from CFD and rises more or less constantly with increasing $b_{2}^{*}$. It was found that, at the high Reynolds numbers encountered here, the surface friction losses in the vaneless space account for less than $8 \%$ of the overall loss, and it is the mixing loss due to the increasing spanwise nonuniform flow that dominates.

Putting it all together, the competing trend in vaneless space and vaned diffuser loss combined with a relatively flat trend in

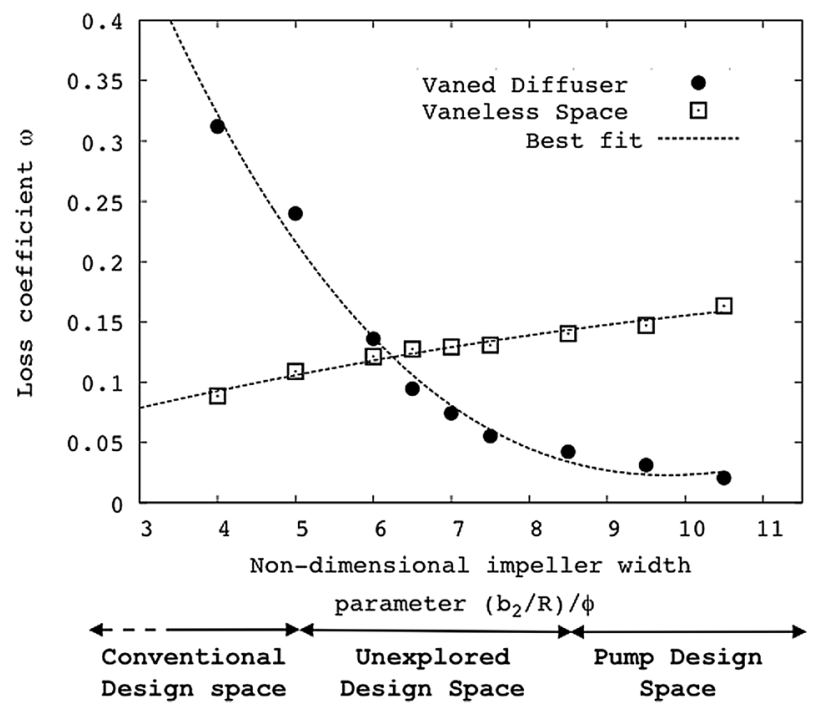

Fig. 4 Effect of $b_{2}^{*}$ on computed vaneless space and estimated vaned diffuser loss coefficients impeller polytropic efficiency and rising exit flow angle suggests that the best overall performance is achieved at a nondimensional impeller width parameter of about 6 . To assess this conjecture, two full stage computations including the vaned diffuser and return channel were carried out for $b_{2}^{*}$ of 6 and 7.5. The simulation results demonstrate that, while the polytropic impeller efficiencies are about the same, the $b_{2}^{*}=7.5$ case yields a one percentage point reduced overall stage efficiency relative to the $b_{2}^{*}=6$ case. This reduction is mainly due to the increased spanwise flow nonuniformity for the wider gas path case, yielding a 1.6 percentage point lower mixed-out averaged impeller efficiency.

In summary, the parametric impeller study suggests that the highest potential efficiency gains can be achieved for a nondimensional impeller width parameter of $b_{2}^{*}=6$. A further increase in impeller width yields higher mixing losses in the stage and nearly tangential flow at impeller exit, reducing the benefits of a wider gas path.

\section{Application: Redesign of Full-Scale Supercritical $\mathrm{CO}_{2}$ Compressors}

The vaned diffuser design concept is first applied to the first stage of the high-pressure compressor block of a full-scale reinjection compressor currently in service. The compressor discharge pressure is $200 \mathrm{bar}$, and the stage under investigation is a low flow coefficient stage with vaneless diffuser operating at supercritical inlet conditions. The second application is the stage redesign of an advanced compressor train with a discharge pressure of 700 bar operating at a lower machine Mach number and with the working fluid close to liquid state. It will be shown that in both cases, significant performance improvements can be achieved when employing a vaned diffuser with a nondimensional width parameter of $b_{2}^{*}=6$.

First, three-dimensional steady, real gas CFD simulations of the datum stage were carried out to compare the computed performance with experimental data and to facilitate the assessment of the design improvements on a consistent basis. Computations were carried out both with and without cavities, seals, and leakage flows. As only flange-to-flange measurements of the high-pressure block were available, the embedded stage performance was deduced from full-machine experiments operating in supercritical $\mathrm{CO}_{2}$ at 200 bar, supported by empirical correlations and data taken from a scaled test rig. In the following, this information is denoted as experimental data.

The computed pressure coefficient and total-to-total polytropic efficiency of the datum stage are compared with experimental data in Figs. 5 and 6. The computations overestimate the measured pressure coefficient by about 0.1 . However, the slope of the pressure rise characteristic is well predicted. The results further suggest that leakage flows yield an efficiency penalty of about 12

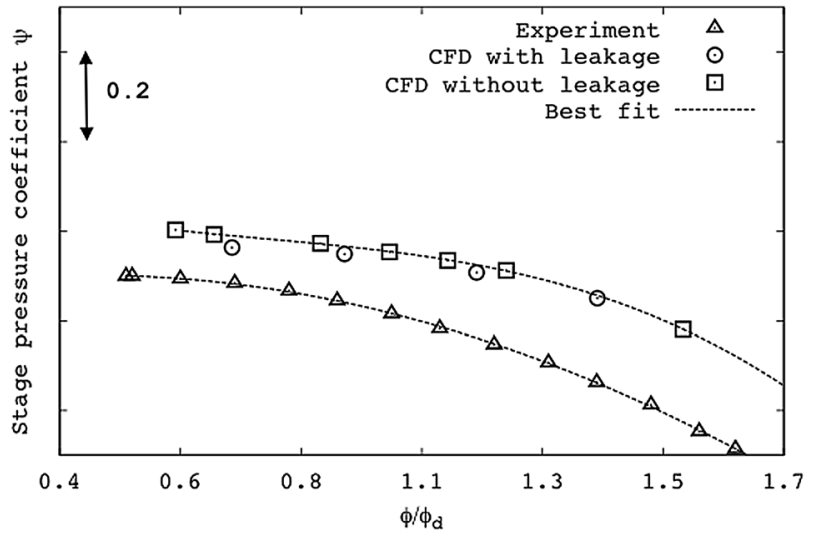

Fig. 5 Assessment of computed datum stage pressure coefficient with experimental data 


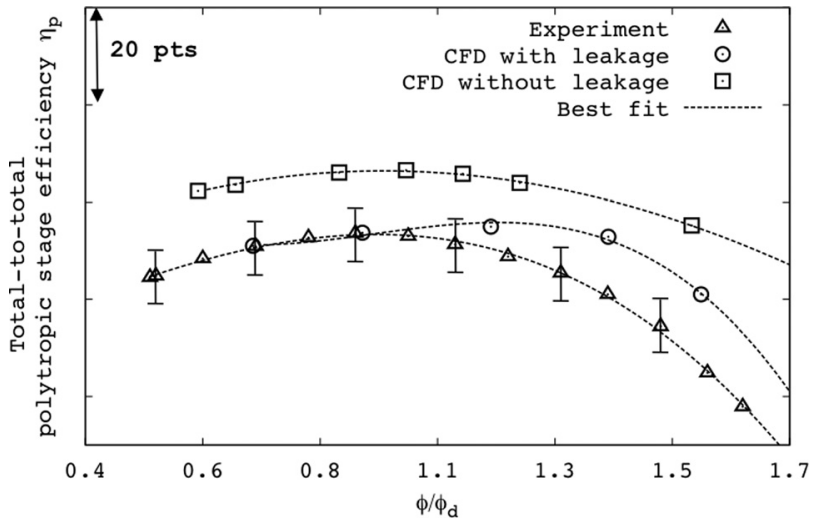

Fig. 6 Assessment of computed datum stage total-to-total polytropic efficiency with experimental data

percentage points. At low flow coefficients, the predicted total-tototal polytropic efficiency is in good agreement with the experimental data while there is an overestimation at high flow coefficients of up to 13 percentage points. This may be due in part to the larger value of the pressure coefficient found in the simulations. The flow coefficient at peak efficiency is well captured in the computations without leakage flow but is overestimated for the case with leakage flow. This is a consequence of the leakage and windage losses, which are more dominant at lower flow coefficients. It is important to note that the experimental data for the stage was estimated from flange-to-flange compressor block measurements by assuming identical losses between the different stages over all flow coefficients. The exact error bands are unknown, but such measurements are unlikely to be better than $\pm 5 \%$ as reported in Fig. 6 .

\section{Compressor Stage Redesign With Vaned Diffuser}

A nondimensional impeller exit width of $b_{2} / R=6 \phi$ is adopted in the stage redesign with vaned diffuser. The pressure coefficient and total-to-total polytropic efficiency of the redesigned stage are compared with the datum design with vaneless diffuser in Figs. 7 and 8 . In this, leakage flows were first neglected to directly assess the impact of the wider impeller and vaned diffuser design strategy. The redesigned stage yields a 0.02 increase in pressure coefficient and a 4.5 percentage point improvement in total-to-total polytropic efficiency relative to the datum stage with vaneless diffuser. At the lowest computed flow coefficient, the slope of the pressure rise coefficient is near zero whereas the characteristic of the datum stage is still negatively sloped. This has implications on

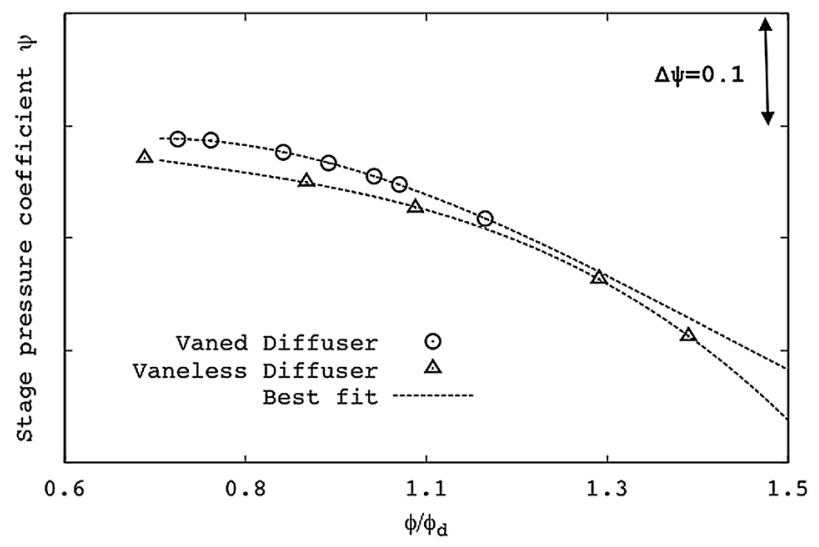

Fig. 7 Computed stage pressure coefficient without leakage flow: wider impeller with vaned diffuser yields 0.02 increase in pressure rise

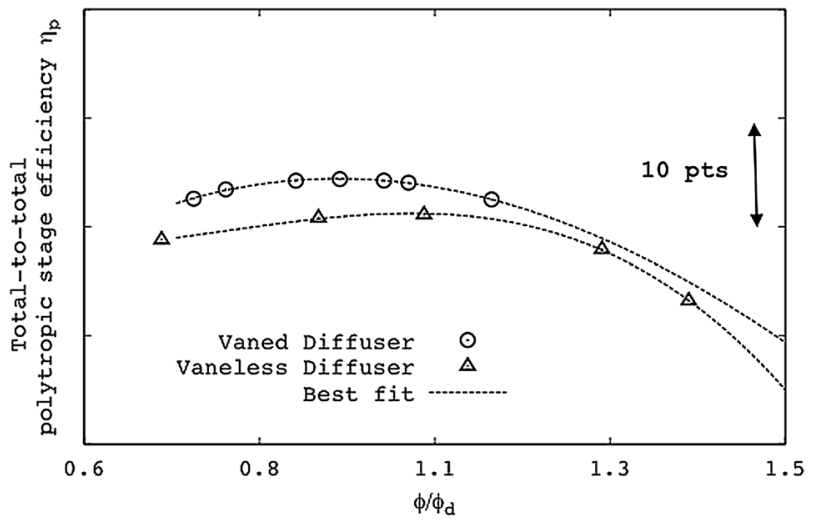

Fig. 8 Computed total-to-total polytropic stage efficiency without leakage flow: wider impeller with vaned diffuser yields a 4.5 percentage point increase in efficiency
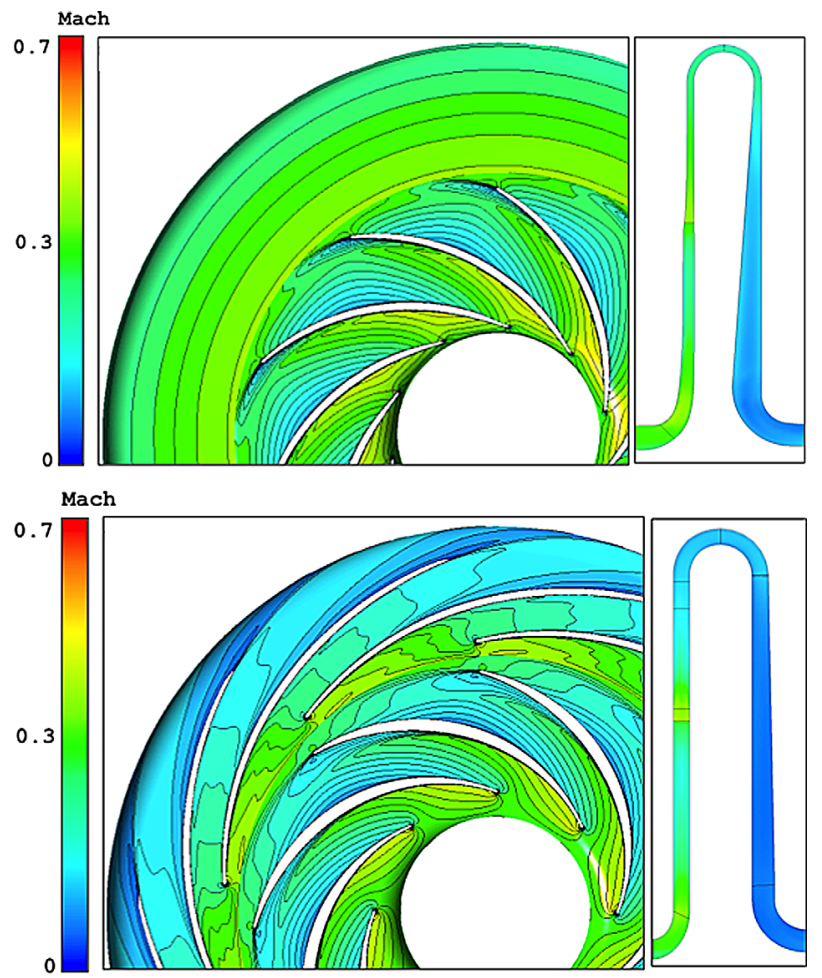

Fig. 9 Absolute Mach number contours at midspan: datum design (top) and redesign (bottom)

compressor stability and dynamic behavior and is discussed in more detail next. The peak efficiency of the redesigned stage occurs near the design flow coefficient suggesting that the stage is well matched.

The comparison of the absolute Mach number field in Fig. 9 reveals that the Mach number at the diffuser exit is nearly half the value of the datum design, reducing end wall losses in the diffuser passage, crossover bend, and return channel.

Next, the redesigned stage performance was assessed including all cavity and leakage flows. The cumulative change in total-tototal polytropic efficiency through the various compressor components is summarized in Fig. 10. Relative to the datum design, the impeller polytropic efficiency is reduced by 1.4 percentage points. This is due in part to increased mixing losses because of the wider gas path but also because of higher leakage losses. As such, the wider impeller yields a higher pressure ratio, which in turn 


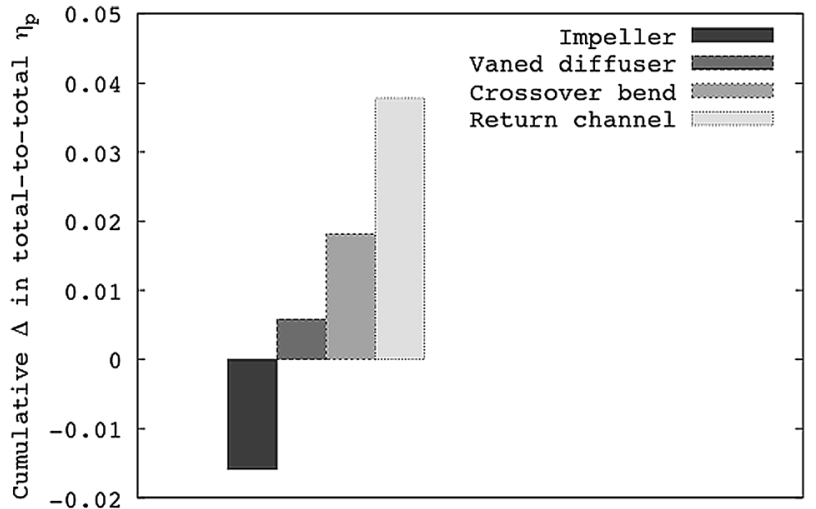

Fig. 10 Cumulative change in total-to-total polytropic efficiency relative to the datum performance

increases the pressure drop across the labyrinth seal. This leads to a larger seal mass flow through the seal, thereby, increasing leakage losses.

To further assess the potential benefit of this design concept, the wider gas path design strategy was also applied to the redesign of an advanced supercritical $\mathrm{CO}_{2}$ reinjection compressor stage with an overall machine discharge pressure of 700 bar. Relative to the previous case, this stage has a higher stage inlet pressure of 200 bar and a lower machine Mach number of 0.4; furthermore, the state of the fluid is closer to that of a liquid. Employing a vaned diffuser enables a radially much more compact machine design but with a pressure recovery similar to that of a vaneless diffuser. This requires the careful design of a low solidity vaned diffuser.

The real gas computations of the redesigned stage were carried out consistent with the previous simulations. Dissection of the component efficiencies shows that the impeller efficiency is nearly three points lower compared to the conventional design due to the higher boss ratio. However, the reduction in friction loss in the diffuser and return channel suggests an efficiency increase of four percentage points in these components. This leads to an overall stage total-to-total polytropic efficiency improvement of one percentage point while maintaining the same pressure rise as the conventional design and increasing the shaft diameter.

\section{Compressor Stability Assessment}

To assess the compressor dynamic behavior and stability, the pressure rise characteristics of the impeller and the vaned diffuser were investigated ${ }^{3}$.

These are plotted in Fig. 11 for the first stage of the highpressure block of the 200 bar compressor train discussed earlier. The steady mixing plane calculations showed small regions of locally reversed flow at corrected flows higher than the expected stall point. This was found to lead to large fluctuations in the pressure coefficient as shown by the error bars in Fig. 11. Unsteady isolated diffuser calculations were, therefore, carried out at the two lowest flow coefficients. The results suggest that the impeller characteristic is negatively sloped throughout the entire operating range whereas the vaned diffuser characteristic turns over at a flow coefficient ratio of 0.8 . This indicates that the overall stability limit of the stage is set by the vaned diffuser.

For the same work coefficient, a wider impeller yields higher diffuser inlet flow angles, potentially reducing the stability margin. Recent work shows that the onset of rotating stall in vaned diffusers is associated with the spanwise nonuniformity of the diffuser inlet flow. More specifically, high incidence near the end wall can cause flow separation and vortex shedding near the

\footnotetext{
${ }^{3}$ The crossover bend and return channel were not included in this assessment as the focus here is on the stability of the impeller and the vaned diffuser.
}

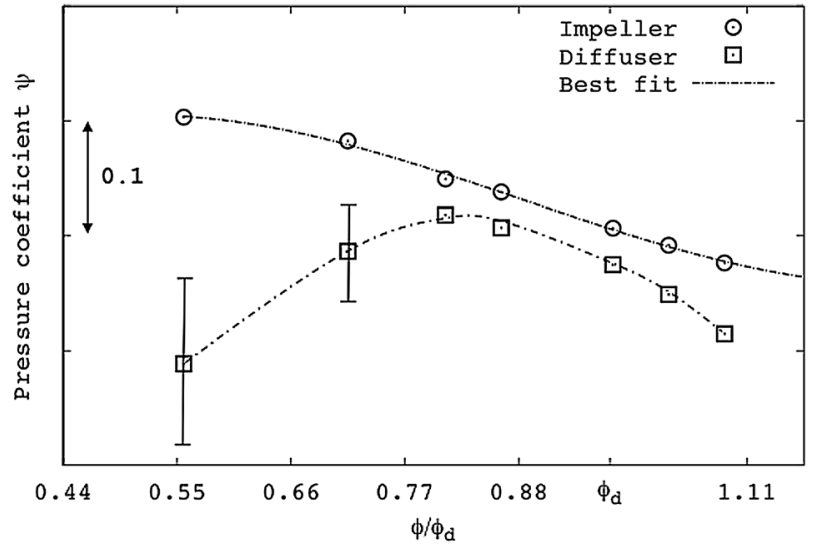

Fig. 11 Impeller and vaned diffuser pressure rise characteristics: diffuser dynamically unstable-error bars indicate fluctuations in pressure rise

diffuser leading edge [11]. This is manifested in the casing static pressure field as short-wavelength stall precursors or so-called "spikes." The low stagnation pressure fluid associated with the shed vorticity convects back into the vaneless space and recirculates around the circumference. As blockage accumulates, the spikes grow and lead to flow breakdown and rotating stall. This mechanism is depicted in Fig. 12.

Common design practice for stability assumes air as the working fluid and sufficient stall margin to avoid potential stability issues. The question arises whether the path into instability and flow conditions at stall onset are possibly altered for very low flow coefficient diffuser designs operating in supercritical $\mathrm{CO}_{2}$. Two important factors that govern the flow angle distribution into the diffuser and, thus, instability onset are rotational effects and viscous effects.

To assess the importance of rotational effects, the ratio of relative flow accelerations to Coriolis accelerations was evaluated. Using the definitions of flow coefficient, the Rossby number $w /$ $\Omega R$ can be written as

$$
R o=\frac{w}{\Omega R}=\frac{\phi R / \cos \beta}{2 \pi b_{2}}
$$

For radial outflow, the Rossby number is directly related to the inverse of the nondimensional impeller width parameter $b_{2}^{*}$. As such, in very low flow coefficient centrifugal compressors where $b_{2}^{*}$ is large, rotational effects are more dominant than inertial effects. Compared to traditional centrifugal compressors, this yields inverted impeller exit flow angle distributions with larger flow angles in the center of the blade passage and reduced swirl near the end walls as shown in Fig. 13. The inverted profile can

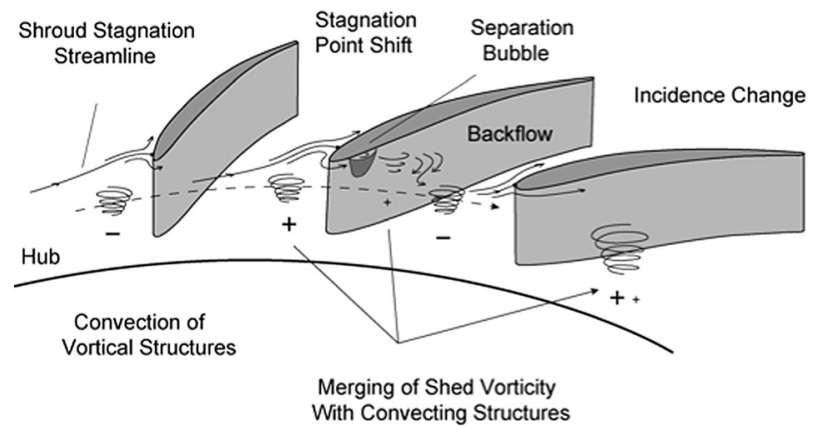

Fig. 12 Mechanism for short-wavelength rotating stall inception in vaned diffusers (adopted from Ref. [11]) 


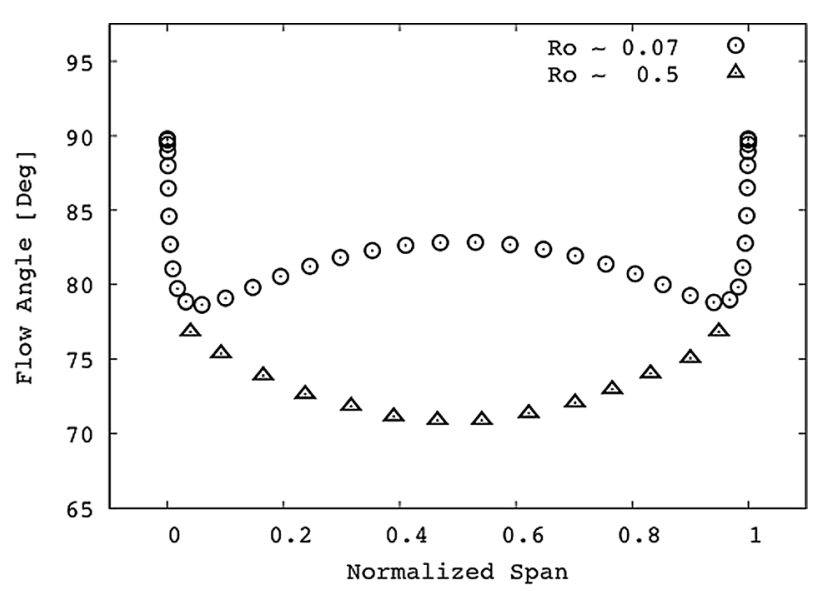

Fig. 13 Spanwise distribution of pitchwise averaged flow angle: typical traditional centrifugal compressor $(R o \sim 0.5)$ versus low flow-coefficient compressor design (Ro $\sim 0.07)$

potentially alter the compressor prestall behavior and stable flow range.

The Reynolds number $\mathrm{Re}=w b_{2} / \nu$ in the supercritical $\mathrm{CO}_{2}$ compressor investigated here is $10^{6}, 2$ orders of magnitude larger than if operating in low-pressure air. As such, viscous effects are confined to very thin layers near the end walls. Combined with the strong rotational effects, the flow field in the impeller is predominantly governed by Coriolis and pressure forces setting up socalled Ekman layers on the end walls as shown in Fig. 14 for low pressure air and supercritical $\mathrm{CO}_{2}$. These are characterized by small Ekman numbers, $E k=\mathrm{Ro} / \mathrm{Re}$ (for more detail on Ekman layers see, for example, Ref. [20]). Note that the Ekman layers in the supercritical $\mathrm{CO}_{2}$ case are much more pronounced, with end wall flow angles reduced by as much as $3 \mathrm{deg}$.

It is, therefore, hypothesized that, for stages where the diffuser limits the stability, the rotating stall onset, more specifically vortex shedding near the diffuser leading edge, occurs at a higher flow coefficient for air than for supercritical $\mathrm{CO}_{2}$. To assess this, unsteady isolated diffuser calculations were carried out with the impeller profile taken from the steady stage calculations starting at flow coefficients where the steady calculations did not converge. For lower flow coefficients, the diffuser inlet flow profiles were taken from steady isolated impeller calculations. The diffuser characteristic for air is plotted as circles in Fig. 15.

Leading edge vortex shedding is first observed at a flow coefficient ratio of 0.81 and is marked by arrow "a." For this operating

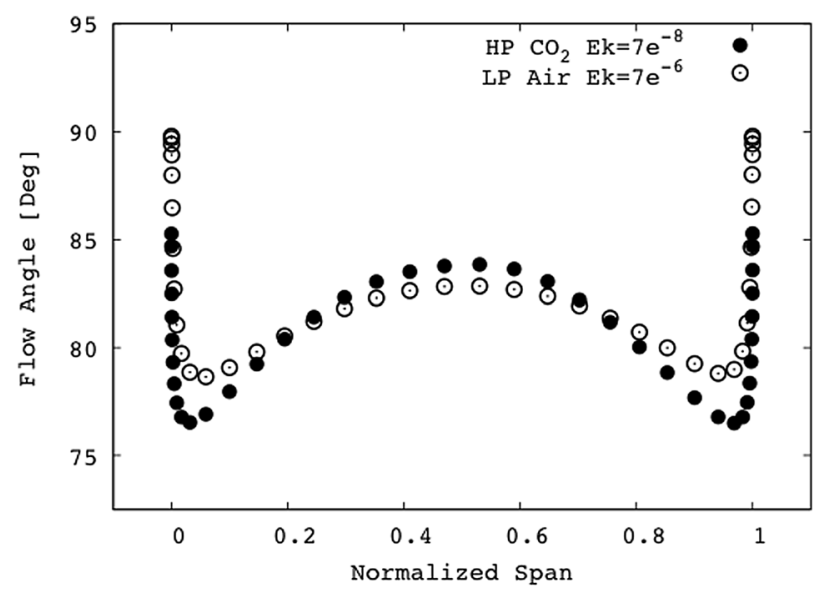

Fig. 14 Ekman layers for low flow-coefficient compressor design. Reduced end wall flow angles for supercritical $\mathrm{CO}_{2}$ suggest increased stability margin relative to low-pressure air.

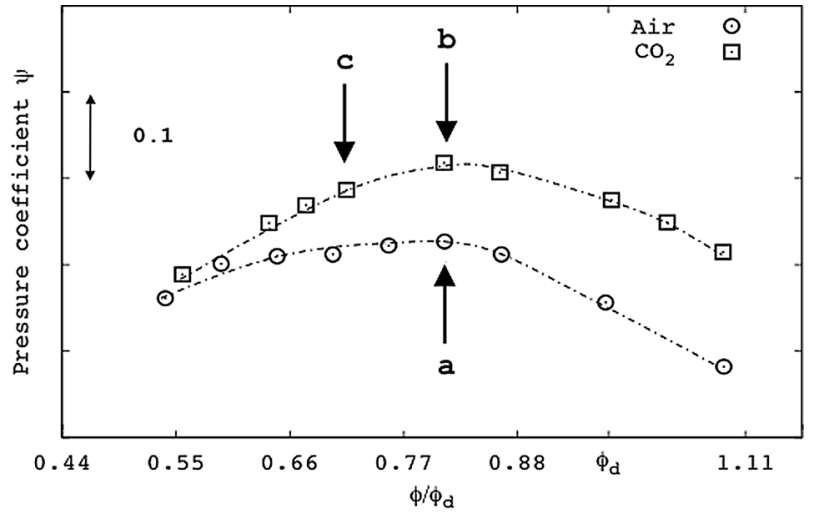

Fig. 15 Diffuser pressure rise characteristics for air and supercritical $\mathrm{CO}_{2}$ : leading edge separation and vortex shedding observed at flow coefficient (a) for air and (c) for supercritical $\mathrm{CO}_{2}$

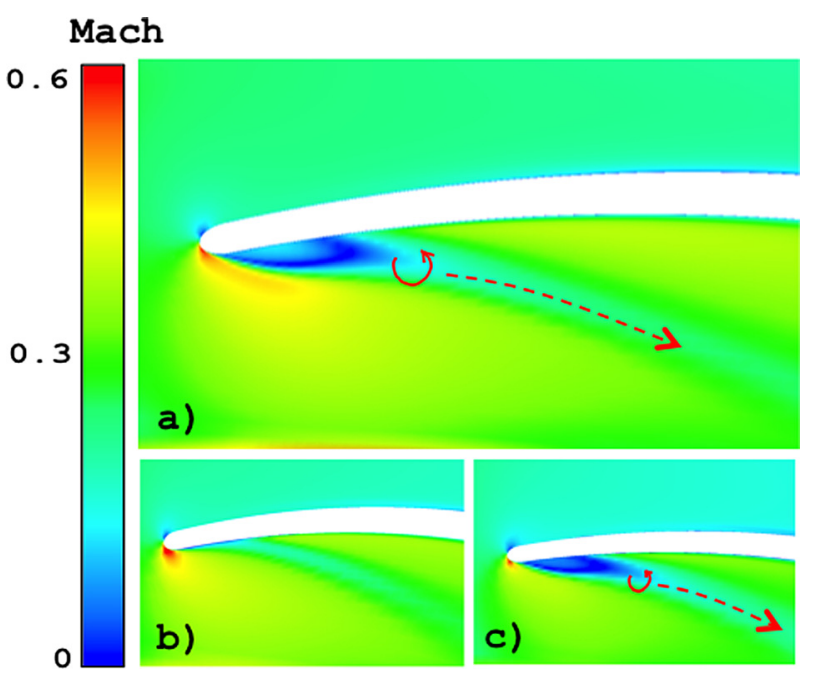

Fig. 16 Mach number contours at $10 \%$ span, (a) air at $\phi /$ $\phi_{d}=0.81$, (b) supercritical $\mathrm{CO}_{2}$ at $\phi / \phi_{d}=0.81$, and (c) supercritical $\mathrm{CO}_{2}$ at $\phi / \phi_{d}=0.68$

point, the instantaneous flow field in the diffuser passage at $10 \%$ span is depicted in Fig. 16(a). The high swirl leads to radially reversed flow in the vaneless space as shown in the Mach number contours. The stagnation point on the pressure side moves further into the passage so that a separation bubble forms near the leading edge on the suction side. Vorticity contours (not shown here) reveal that this low stagnation pressure fluid is shed as a vortex, which convects tangentially and radially inward and disturbs the flow into the neighboring diffuser passage. The vortex shedding extends from the hub all the way to about $40 \%$ of span, consistent with the swirl angle distribution depicted in Fig. 17, where the case for air is marked by the open circles.

Next, unsteady isolated diffuser calculations were carried out in supercritical $\mathrm{CO}_{2}$, starting at the operating condition where vortex shedding was first observed in air, indicated by arrow "b" in Fig. 15. At this flow coefficient, a small separation bubble forms near the leading edge but the flow reattaches. The flow stays attached along the entire span as illustrated by the instantaneous flow field in Fig. 16(b) with vortex shedding absent.

As the flow coefficient is further reduced, the stagnation point moves along the pressure side into the passage, and vortex shedding is initiated at a flow coefficient ratio of 0.68 . This occurs at a flow coefficient ratio less than that in air and is marked by arrow "c" in Fig. 15. Similar to the case in air, the flow angle profile is 


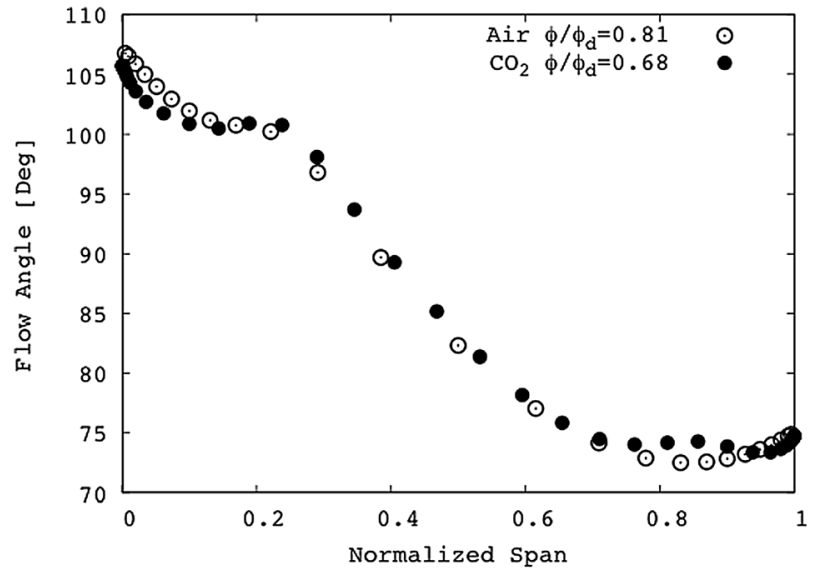

Fig. 17 Spanwise distribution of pitchwise averaged flow angle for air and supercritical $\mathrm{CO}_{2}$ at onset of leading edge vortex shedding

skewed with higher angles near the hub as shown by the full circles in Fig. 17. As a consequence, vortex shedding occurs as shown in the instantaneous Mach number field in Fig. 16(c).

Returning to Fig. 16(c), the unsteady flow is qualitatively the same as in Fig. 16(a): Vortices are shed from the leading edge and convect around the circumference. This behavior is consistent with the mechanism reported in Ref. [11] and illustrated in Fig. 13 , suggesting that the path into instability is governed by the formation of spikes. Full annulus unsteady calculations or a highly instrumented diffuser experiment would be required to fully validate this conjecture. This is the objective of ongoing and future work.

In summary, the inverse of the nondimensional impeller exit width parameter $b_{2}^{*}$ can be interpreted as the Rossby number. In very low flow coefficient compressor designs, the Coriolis accelerations dominate the relative flow accelerations, which lead to inverted swirl angle distributions at the impeller exit. Combined with the 2 orders of magnitude higher Reynolds number for supercritical $\mathrm{CO}_{2}$, the leading edge vortex shedding occurs at lower flow coefficients than in air. The computations indicate that this can lead to a difference in stall margin of up to 11 points, adding extra margin for supercritical $\mathrm{CO}_{2}$ and deeming the design intent based on air conservative.

\section{Conclusions}

Multistage compressors operating with supercritical $\mathrm{CO}_{2}$ for CCS and EOR applications require stages with a relatively thick shaft, a wide operating range, and need to have good efficiency while operating at very low flow coefficients. This paper examined a design strategy for such stages, which involved an increase in the impeller width and the use of a vaned diffuser. CFD simulations of several stages were undertaken, including a parametric study of the effect of a change in the impeller outlet width. The simulations indicate that the best efficiency improvement with this strategy occurs with a nondimensional impeller outlet width parameter of $b_{2}^{*}=\left(b_{2} / R\right) / \varphi=6$ and that this optimum is governed by a balance of mixing and friction losses.

The design strategy was first applied to a full-scale reinjection compressor currently in service. Three-dimensional, steady, supercritical $\mathrm{CO}_{2} \mathrm{CFD}$ simulations of the full stage with leakage flows were carried out with the NIST real gas model. The design study suggests that a nondimensional width parameter of $b_{2}^{*}=6$ yields a 3.5 point increase in efficiency relative to that of a traditional compressor design with vaneless diffuser. In order to meet the requirements of a thicker shaft, some of this efficiency is then sacrificed in the interests of improved rotordynamics. With this additional constraint, the redesign of an advanced compressor stage for a 700 bar application yields an efficiency improvement of about $1 \%$.

The new stage design was also examined with respect to its operating range. The unsteady computations indicate that the vaned diffuser limits the stability of the stage and that the onset of stall is likely caused by vortex shedding near the diffuser leading edge. The investigation further suggests that, governed by more pronounced Ekman layers for supercritical $\mathrm{CO}_{2}$, this occurs at lower flow coefficients than in air.

\section{Acknowledgment}

This research was funded by Mitsubishi Heavy Industries Takasago R\&D Center, which is gratefully acknowledged. In particular, the authors would like to thank Dr. Sumiu Uchida, Dr. Eisaku Ito, and Mr. Akihiro Nakaniwa for their support and for providing the experimental data. PCA Engineers Ltd. are also thanked for the use of their Vista radial compressor design software for this work.

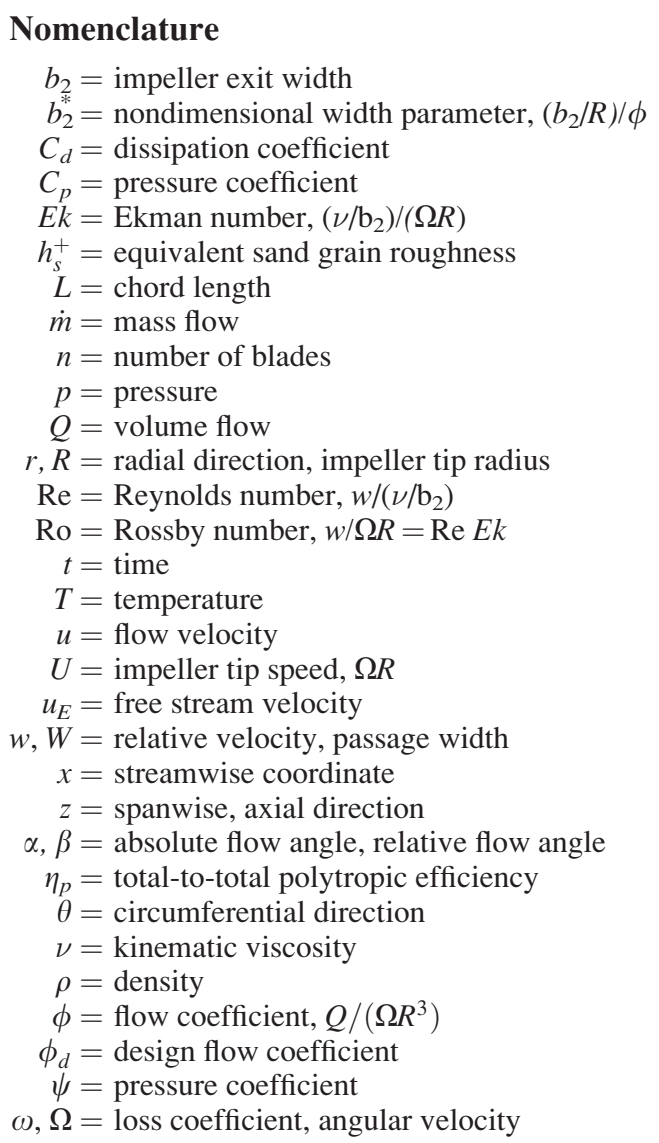

\section{References}

[1] Smith, R., 2009, "U.S. EPA: Report on the Environment," Technical Report, U.S Environmental Protection Agency, Washington, DC.

[2] Kobayashi, S., and Van Hassel, B., 2005, " $\mathrm{CO}_{2}$ Reduction by Oxy-Fuel Combustion: Economics and Opportunities," GCEP Advanced Coal Workshop, Provo, UT, March 15-16

[3] Aungier, R. H., 2000, Centrifugal Compressors: A Strategy for Aerodynamic Design and Analysis, ASME Press, New York.

[4] Cumpsty, N. A., 1989, Compressor Aerodynamics, Longman, London.

[5] Gülich, J. F., 2003, "Disk Friction Losses of Closed Turbomachine Impellers," Forsch. Ingenieurwes., 68, pp. 87-95.

[6] Lüdtke, K. H., 2004, Process Centrifugal Compressors, Springer, Berlin.

[7] Casey, M. V., Dalbert, P., and Schurter, E., 1990, "Radial Compressor Stages for Low Flow Coefficients," 4th European Congress on Fluid Machinery for Oil, Petrochemical and Related Industries, The Hague, Netherlands, May 21-23, IMechE Paper No. C403/004.

[8] Dalbert, P., Ribi, B., and Casey, M. V., 1999, "Radial Compressor Design for Industrial Compressors,” Proc. Inst. Mech. Eng., Part C: Mech. Eng. Sci., 213, pp. 71-83. 
[9] Gülich, J. F., 2008, Centrifugal Pumps, Springer, Berlin.

[10] Everitt, J. N., and Spakovszky, Z. S., 2013, "An Investigation of Stall Inception in Centrifugal Compressor Vaned Diffuser," ASME J. Turbomach., 135(1), p. 011025 .

[11] Senoo, Y., Kinoshita, Y., and Masahiro, I., 1977, "Asymmetric Flow in Vaneless Diffusers of Centrifugal Blowers," ASME J. Fluids Eng., 99(1), pp. 104-111.

[12] Japikse, D., 1994, Centrifugal Compressors Design and Performance, Concepts ETI, White River Junction, VT.

[13] Casey, M. V., and Robinson, C. J., 2010, "A New Streamline Curvature Throughflow Code for Radial Turbomachinery," ASME J. Turbomach., 132(3), p. 031021.

[14] Casey, M. V., 1983, "A Computational Geometry for the Blades and Internal Flow Channels of Centrifugal Compressors," ASME J. Eng. Power, 105(2), pp. 288-295.
[15] Ansys, 2012, "Academic Research, Release 14.5, Theory Manual," Ansys, Inc., Irvine, CA.

[16] Lemmon, E. W., Huber, M. L., and McLinden, M. O., 2010, "NIST Reference Fluid Thermodynamic and Transport Properties-REFPROP, Version 9.0," NIST Standard Reference Database 23, National Institute of Standards and Technology, Gaithersburg, MD.

[17] Span, R., and Wagner, W., 1996, "A New Equation of State for Carbon Dioxide Covering the Fluid Region From the Triple Point Temperature to $1100 \mathrm{~K}$ at Pressures Up to 800 MPa," J. Phys. Chem. Ref. Data, 25(6), pp. 1509-1596.

[18] Schultz, J., 1962, "The Polytropic Analysis of Centrifugal Compressors," ASME J. Eng. Power, 12, pp. 69-89.

[19] Denton, J. D., 1993, "Loss Mechanisms in Turbomachines," ASME J. Turbomach., 115(4), pp. 621-656.

[20] Greitzer, E. M., Tan, C. S., and Graf, M. B., 2004, Internal Flow Concept and Applications, Cambridge University, Cambridge, UK. 